\section{Response to Johansen Taber et al.}

\section{To the Editor:}

We appreciate the interest by Myriad Women's Health in our recent study on the adherence by commercial companies to the American College of Medical Genetics and Genomics (ACMG) recommendations on cell-free DNA (cfDNA) noninvasive prenatal screens (NIPS). ${ }^{1,2}$ We are eager to update our assessments with any new information, and we welcome direct contact and dialogue from NIPS companies. Indeed, we have already received revised materials from at least one lab. As indicated in our paper, the latest version of Table 2 from our study is posted at https:// prenatalinformation.org/table/, reflective of any new supporting documentation that has been received from NIPS companies.

The documentation we used for our analyses is itemized in the Supplementary Materials to our paper. For Counsyl's Prelude (now Myriad's Prequel) NIPS test, this included analyzing patient and provider material posted on their web page and provided at their exhibitors' booth during the 2018 ACMG meeting. We also analyzed sample reports provided directly to us from Counsyl. (To be objective and consistent in our methodology, we directly reached out to all of the NIPS laboratories to obtain any missing sample reports that could not be found on their web pages.) To be ecologically valid, we sought to mimic information requests from ordering physicians and/or expectant parents. After much discussion, we chose to analyze those companies that had the largest market share of the NIPS tests commercially available in the United States as of 1 January 2018. We agree that NIPS tests from single health systems, single-hospital systems, and academic/university settings should also be analyzed, and we hope to do so in future research. We also agree that additional studies should examine why laboratories are not following certain ACMG recommendations.

The authors noted three primary concerns. First, we applaud their efforts in including detection rate (DR), specificity (SPEC), positive predictive value (PPV) (patientspecific), and negative predictive value (NPV) for common aneuploidies on their test reports. We chose to give Counsyl a yellow (or partial adherence) to recommendation 1 because not all pretest marketing materials had the same consistent information. We sought to be as objective as possible, simply looking for adherence to the ACMG recommendations as they were written. For recommendation 8, we gave Counsyl a red (or little to no adherence) because Counsyl wrote on sample reports for some sex aneuploidies that "PPV... cannot be calculated due to insufficient prevalence data." The ACMG guidelines recommend that "[1]aboratories provide modeled PPV when reporting positive results for which neither patient-specific nor population-derived PPV are possible."3

Second, the authors state that they "do not believe it is clinically practical or responsible" to include the data requested by ACMG in recommendation 7. The ACMG position statement allows the use of modeled data when laboratory-specific data are not available. ${ }^{3}$ The statement further says that "when laboratories cannot report specific DR, SPEC, PPV, and NPV, screening for those CNVs should not be performed by that laboratory." 3 We appreciate that these authors did not have disagreement with our assessment, per se, but rather with this ACMG recommendation in general. We defer to the ACMG on when their position statement on NIPS might be updated again.

Third, we acknowledge the good faith efforts by Myriad to provide information about patient resources in their test reports. When assessing the provision of patient education resources, we constructed a matrix of the five recommended patient education resources and the five provider resources mentioned in the ACMG guidelines. ${ }^{3}$ If a lab listed any of one of these resources on their lab reports or publicly available websites or patient education pamphlets, then we assigned a yellow rating for making a good faith effort. A red rating meant the labs provided none of the recommended resources in any publicly available medium or lab reports. A company was rated a green score if they listed at least 3 of 5 recommended patient and provider resources. Myriad did offer one of the recommended patient education resources in multiple mediums, as well as references to additional valuable educational resources beyond the recommendations, but they were missing all the recommended provider resources.

The responsible clinical implementation of cfDNA NIPS tests requires cooperation and collaboration from labs, clinicians, insurers, professional societies, and patients alike. Commercial labs are vital partners who have a direct impact on every patient undergoing testing. They also remain the greatest source of funding for materials to support those patients. Through adherence to the ACMG recommendations, we hope that labs will help ensure that providers are getting all the information they need about testing and that vulnerable families are not left isolated and confused about powerful genetic information.

\section{DISCLOSURE}

The conflicts for the authors remain unchanged from those declared in our original publication. Financial conflicts of interest: B.G.S. occasionally consults on the topic of Down syndrome (DS) through Gerson Lehrman Group. He receives remuneration from DS nonprofit organizations for speaking engagements and 
associated travel expenses. B.G.S. receives annual royalties from Woodbine House, Inc., for the publication of his book, Fasten Your Seatbelt: A Crash Course on Down Syndrome for Brothers and Sisters. Within the past 2 years, B.G.S. has received research funding from F. Hoffmann-La Roche, Inc. to conduct clinical trials on study drugs for people with DS. B.G.S. is occasionally asked to serve as an expert witness for legal cases where DS is discussed. M.W.L. and S.M. are employees of the National Center for Prenatal \& Postnatal Resources at the University of Kentucky, which produces and sells patient educational material recommended by professional guidelines; M.W.L. and S.M. receive no direct compensation from the sale of such materials. K.B. has financial holdings in GenomeSmart, a patient education platform. M.A.A. is an employee of Mayo Clinic, which provides cell-free DNA testing services through Mayo Medical Laboratories; M.A.A. receives no direct compensation from the sale of such tests. Other conflicts of interest: Beyond the items mentioned in the financial disclosures above, B.G.S. serves in a nonpaid capacity on the Honorary Board of Directors for the Massachusetts Down Syndrome Congress, the Board of Directors for the Band of Angels Foundation, and the Professional Advisory Committee for the National Center for Prenatal and Postnatal Down Syndrome Resources. B.G.S. has a sister with DS. M.W.L. serves in a nonpaid capacity on the D.S. Education Foundation for Down Syndrome of Louisville. M.W.L. has a daughter with DS. S.M. has a son with DS. M.A.A. has received travel funding compensation from the Association for $X$ and $Y$ Chromosome Variations. The other authors declare no conflicts of interest.
Publisher's note: Springer Nature remains neutral with regard to jurisdictional claims in published maps and institutional affiliations.

Brian G. Skotko, MD, MPP(D ${ }^{1,2}$, Megan A. Allyse, $P h D^{3}$, Komal Bajaj, MD, MS-HPEd ${ }^{4}$, Robert G. Best, PhD ${ }^{5}$, Mark Leach, JD, $M A^{6}$, Stephanie Meredith, $M A^{6}$, Marsha Michie, $\mathrm{PhD}$ (D) $^{7}$ and Katie Stoll, $M S^{8}$

${ }^{1}$ Division of Medical Genetics, Department of Pediatrics, Massachusetts General Hospital, Boston, MA, USA; ${ }^{2}$ Department of Pediatrics, Harvard Medical School, Boston, MA, USA; ${ }^{3}$ Program in Biomedical Ethics Research and Department of Obstetrics and Gynecology, Mayo Clinic, Rochester, MN, USA; ${ }^{4}$ Division of Reproductive and Medical Genetics, Department of Obstetrics \& Gynecology and Women's Health, Montefiore Medical Center/Albert Einstein College of Medicine, Bronx, NY, USA; ${ }^{5}$ University of South Carolina School of Medicine Greenville/ Greenville Health System, Greenville, SC, USA; ${ }^{6}$ National Center for Prenatal \& Postnatal Resources, University of Kentucky, Lexington, KY, USA; ${ }^{7}$ Department of Bioethics, Case Western Reserve University School of Medicine, Cleveland, OH, USA; ${ }^{8}$ Genetic Support Foundation, Olympia, WA, USA. Correspondence:

Brian G. Skotko (bskotko@mgh.harvard.edu)

\section{REFERENCES}

1. Taber J. Inaccuracies and shortcomings in "Adherence of cell-free DNA noninvasive prenatal screens to ACMG recommendations". Genet Med. 2019. https://doi.org/10.1038/s41436-019-0555-5.

2. Skotko BG, et al. Adherence of cell-free DNA noninvasive prenatal screens to ACMG recommendations. Genet Med. 2019. https://doi.org/ 10.1038/s41436-019-0485-2 [Epub ahead of print].

3. Gregg $A R$, et al. Noninvasive prenatal screening for fetal aneuploidy, 2016 update: a position statement of the American College of Medical Genetics and Genomics. Genet Med. 2016;18:1056-1065.

Advance online publication 3 June 2019. doi:10.1038/s41436-019-0556-4 